\title{
Expression pattern of DKK3, dickkopf WNT signaling pathway inhibitor 3 , in the malignant progression of oral submucous fibrosis
}

\author{
SHANGHUI ZHOU ${ }^{1}$, YUN ZHU ${ }^{1}$, MUBARAK MASHRAH ${ }^{1}$, XINYU ZHANG $^{1}$, ZHIJING HE $^{2}$, \\ ZHIGANG YAO ${ }^{3}$, CHUNYE ZHANG $^{4}$, FENG GUO ${ }^{5}$, YONGJIE HU ${ }^{1}$ and CHENPING ZHANG ${ }^{1}$ \\ ${ }^{1}$ Department of Oral and Maxillofacial - Head and Neck Oncology, Shanghai Ninth People's Hospital, \\ Shanghai Jiaotong University School of Medicine, Shanghai 200011; ${ }^{2}$ Department of Oral and Maxillofacial Surgery, \\ The Second Xiangya Hospital, Central South University, Changsha, Hunan 410011; ${ }^{3}$ Department of Oral Pathology, \\ Xiangya Stomatological Hospital, Central South University, Changsha, Hunan 410078; ${ }^{4}$ Department of Oral Pathology, \\ Shanghai Ninth People's Hospital, Shanghai Jiaotong University School of Medicine, Shanghai 200011; \\ ${ }^{5}$ Department of Oral and Maxillofacial Surgery, Xiangya Hospital, Central South University, \\ Changsha, Hunan 410008, P.R. China
}

Received April 29, 2016; Accepted June 11, 2016

DOI: $10.3892 / o r .2016 .5307$

\begin{abstract}
Oral squamous cell carcinoma (OSCC) is a common malignant neoplasm of the head and neck. Oral submucous fibrosis (OSF) is one of its pre-cancerous lesions; however, the key molecular events in the tumorigenesis of OSF remain elusive. Dickkopf WNT signaling pathway inhibitor 3 (DKK3) is one of the Wnt antagonists, and its downregulation and methylation have been reported in multiple malignancies, while no report of its expression in the carcinogenesis of OSF exists. In the present study, we investigated DKK3 expression at the protein and mRNA levels by immunochemical staining and semi-quantitative RT-PCR in normal oral, OSF and OSCC tissues. We found that DKK3 was readily expressed in normal oral mucous tissues, but was gradually increased in early, moderately advanced and advanced OSF tissues, and strongly expressed in OSCC tissues. DKK3 was localized in the cytoplasm during OSF progression. A rare mutation of DKK3 was observed in OSCC, along with increased copy numbers. Furthermore, through analysis of its co-expressed genes, DDK3 may deregulate Wnt signaling, p53 signaling, apoptosis, $\mathrm{Ca}^{2+}$ signaling and mitochondrial signaling pathways in OSCC pathogenesis. Thus, our results demonstrated that DKK3 is upregulated in the carcinogenesis of OSF, due to gain of copy number, which could be a potential tumor marker for the early detection of OSCC.
\end{abstract}

Correspondence to: Professor Chenping Zhang, Department of Oral and Maxillofacial - Head and Neck Oncology, Shanghai Ninth People's Hospital, Shanghai Jiaotong University School of Medicine, 639 Zhizaoju Road, Huangpu, Shanghai 200011, P.R. China E-mail: zhang.chenping@hotmail.com

Key words: DKK3, OSF, OSCC, tumor marker, early detection

\section{Introduction}

Oral squamous cell carcinoma (OSCC) constitutes more than $90 \%$ of all malignant neoplasms in the oral cavity, with an increased number of new OSCC cases worldwide and varied geographical distribution $(1,2)$. OSCC is prevalent in Southern Asia and Latin America, and is the most common cancer among the male population $(3,4)$. In the high-risk areas, betel quid (BQ) chewing, tobacco and alcohol consumption have been well documented as etiologic factors for $\operatorname{OSCC}(4,5)$. A causal relationship between $\mathrm{BQ}$ chewing and the high incidence of oral cancer has been established, particularly its correlation with early oral premalignancies such as oral submucous fibrosis (OSF) (6,7). Despite the recent rapid improvement in multimodality treatment, the overall 5-year survival rate of OSCC remains poor. Thus, elucidation of molecular events during OSCC development and progression is needed for the early detection and therapy of OSCC.

Overactivation of $\mathrm{Wnt} / \beta$-catenin signaling has been identified in the tumorigenesis of multiple cancers including OSCC, and plays critical roles in cell proliferation, differentiation, adhesion and stemness (7,8). Wnt ligands, antagonists and mutations of APC and $\beta$-catenin have been well-defined for its activation. The dickkopf (DKK) family as Wnt antagonists, contains five members, DKK1, DKK2, DKK3, DKK4 and DKKL1, with different impacts on $\mathrm{Wnt} / \beta$-catenin signaling $(9,10)$. However, the underlying mechanisms remain elusive. Notably, the secreted Wnt antagonist dickkopf-related protein 3 (DKK3) is emerging as a crucial regulator of human cancers (9). DKK3 is frequently reduced by promoter methylation in multiple solid and hematological cancers, acting as a functional tumor suppressor by affecting apoptosis and proliferation. Re-introduction of DKK3 expression in cancer cells may ba a potential biomarker and effective therapeutic approach. 
There are several contradictory studies of DKK 3 in head and neck squamous cell carcinomas (HNSCC) and OSCC. Its tumor suppressive and oncogenic functions have been reported in OSCC (11-15). However, the expression pattern of DKK3 in the malignancy progression of OSF has not been addressed. In the present study, we examined DKK3 expression at the protein and mRNA levels in normal oral mucosa, OSF and OSCC tissues, as well as the correlation with clinicopathological features. We also analyzed its potential function in the pathogenesis of OSCC.

\section{Materials and methods}

Tissue specimens. OSCC $(\mathrm{n}=55), \mathrm{OSF}(\mathrm{n}=45)$, and normal oral mucosa $(n=15)$ tissue specimens were obtained at the time of surgical resection at Xiangya Second Hospital and Xiangya Hospital, Central South University (Changsha, China) and Shanghai Ninth People's Hospital, Shanghai Jiaotong University School of Medicine (Shanghai, China) from January 2013 to June 2014. The patient informed consents were obtained under a protocol reviewed and approved by the Institutional Review Boards of the Xiangya School of Medicine or Shanghai Jiaotong University School of Medicine. The clinical diagnosis and pathologic stage of the OSF were determined in terms of the Pingborg criteria (16). OSCC was diagnosed according to the World Health Organization criteria of 2005. Fifteen normal specimens were obtained from healthy oral mucosa. Forty-five cases of OSF were incident, newly diagnosed without OSCC or neoplastic disease. OSF was classified into 3 grades: early stage $(E, n=15)$, moderately advanced stage $(M$, $\mathrm{n}=15)$ and advanced stage $(\mathrm{A}, \mathrm{n}=15)$. All collected tissues were divided into two parts, one of which was frozen immediately at $-80^{\circ} \mathrm{C}$ after careful removal of the tumor mass, OSF tissue in the epithelium layer and grossly normal-looking epithelium, and the other part was fixed in $4 \%$ buffered formalin solution for pathologic diagnosis and immunohistochemical staining. Clinicopathologic staging of OSCC was determined by the TNM classification of the International Union Against Cancer in 2009. The results of the immunostained formalin-fixed, paraffin-embedded (FFPE) sections were evaluated separately by two pathologists (C.Z. and Z.Y. at the Department of Oral Pathology of the respective affiliate hospital).

Immunohistochemistry. Immunohistochemical staining was performed on $4-\mu \mathrm{m}$ serial sections from FFPE specimens. After deparaffinization and hydration, the slides were treated with endogeneous peroxidase in $3 \% \mathrm{H}_{2} \mathrm{O}_{2}$ for $20 \mathrm{~min}$. The sections were then blocked for $30 \mathrm{~min}$ at $37^{\circ} \mathrm{C}$ with $1.5 \%$ blocking serum in phosphate-buffered saline (PBS) before reacting with DKK3 (1:100 dilution; \#10365-1-AP; ProteinTech) at $4^{\circ} \mathrm{C}$ in a moist chamber overnight. Negative control slides were duplicate sections in the absence of primary antibodies. For evaluating DKK3 expression, a scoring method was used. A mean percentage of positive cells was determined by the examination of 500 cells in at least 5 areas at a magnification of $x 400$. Cells were assigned to 1 of the 5 following categories according to the percentage of positive cells (PP): $0,<5 \%$; $1,5-24 \% ; 2,25-49 \% ; 3,50-75 \%$; and $4,>75 \%$. The intensity of the DKK3 staining (SI) was then scored as follows: 0 , negative (-); 1 , weak (+); 2 , moderate (++); and 3, intense (+++). The final immunoreactive score (IRS $=$ SI + PP) was as follows:,- 0 or $1 ;+, 2$ or $3 ;++, 4$ or 5 ; and,+++ 6 or 7 . The stained tissues were scored blindly regarding clinical patient data. Statistical analyses were performed with SPSS 19.0 software. Statistical significance was evaluated by the Chi-square test $\left(\chi^{2}\right)$. The significance level was set at 0.05 .

Reverse-transcription polymerase chain reaction (RT-PCR). TRIzol reagent (Invitrogen Life Technologies, Karlsruhe, Germany) was used to extract total RNAs. Reverse transcription polymerase chain reaction (RT-PCR) was performed using a kit from Promega (Madison, WI, USA). Real-time PCR was performed to detect $D K K 3$ expression, according to the manufacturer's protocol (HT7500 system; Applied Biosystems). Primers for amplifying $D K K 3$ mRNA sequences were synthesized as previously described. The 161-bp mRNA of $D K K 3$ was amplified by PCR with forward primer, 5'-CAC CCTCAATGAGATGTTCC and reverse primer, 5'-TGGTCTC ATTGTGATAGCTG. GAPDH as a control for all the samples was shown in our previous studies $(17,18)$. PCR amplification was performed with denaturation at $94^{\circ} \mathrm{C}$ for $30 \mathrm{sec}$, annealing at $55^{\circ} \mathrm{C}$ for $30 \mathrm{sec}$, and extension at $72^{\circ} \mathrm{C}$ for $30 \mathrm{sec}$ in 32 cycles. The PCR products were visualized on $2 \%$ agarose gels under ultraviolet transillumination.

\section{Results}

Increased expression levels of DKK3 in OSCC and HNSCC. We firstly investigated $D K K 3$ expression in OSCC and HNSCC using the open access cancer microarray database Oncomine (https://www.oncomine.org/resource/login.html). Through analysis of studies by Peng et al, Ye et al and Ginos et al, the results revealed that $D K K 3$ expression at the mRNA level was 1.377-fold higher in OSCC than that in normal oral mucosa tissues ( $\mathrm{P}=0.002)(19), 1.433$-fold higher in tongue squamous cell carcinoma (TSCC) tissues than that in normal tongue mucosa tissues ( $\mathrm{P}=2.63 \mathrm{E}-4)(20), 1.831$-fold higher in HNSCC tissues than that in normal buccal mucosa ( $\mathrm{P}=1.93 \mathrm{E}-5)$ (21) (Fig. 1A-C). By analyzing microarray data from the GENT dataset (http:// medical-genome.kribb.re.kr/GENT/search/search.php), DKK3 was found to be overexpressed in HNSCC tissues, compared with the level in the corresponding normal oral mucosa tissues (Fig. 1D). These results suggest that DKK3 upregulation may play a crucial role in OSCC development.

Genetic alteration of DKK3 in OSCC. We next assessed genetic alterations of DKK3 in HNSCC including OSCC using The Cancer Genome Atlas (TCGA) database. DKK3 was overexpressed in two TCGA HNSCC cohorts, with a rare mutation (Fig. 2A). Copy number analysis using Oncomine database showed an increased $D K K 3$ gene copy number in OSCC tissues $(\mathrm{P}=1.75 \mathrm{E}-4)$, compared with that noted in normal oral mucosa tissues (Fig. 2B). Although deletion of $D K K 3$ was observed in the HNSCC TCGA cohort, there was no statistically significant difference (Fig. 2C). These results suggest that gain of $D K K 3$ gene copy number may lead to increased $D K K 3$ expression level in OSCC.

DKK3 upregulation at the protein level in the carcinogenesis of $O S F$. To evaluate DKK3 protein expression in malignant progression of OSF, we firstly performed immunohistochemical 
A

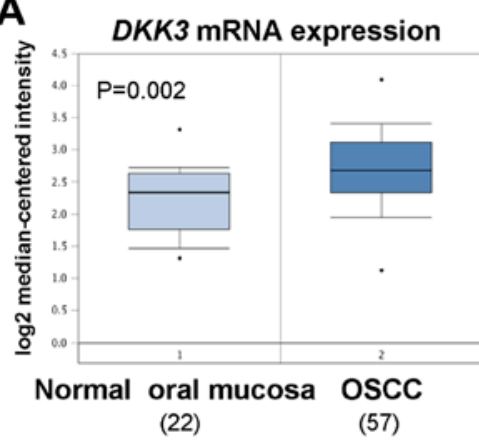

C DKK3 mRNA expression

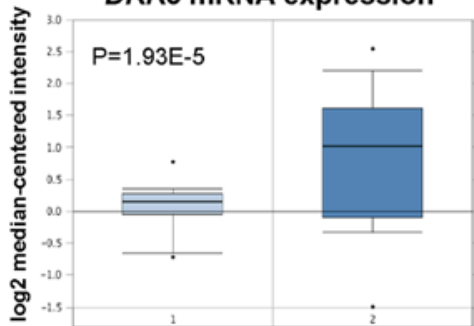

Normal buccal mucosa HNSCC

(13)
B

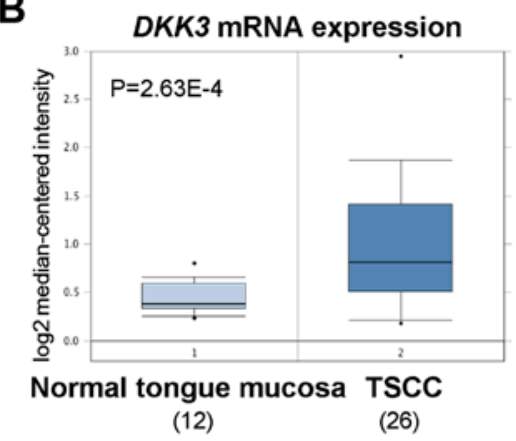

D

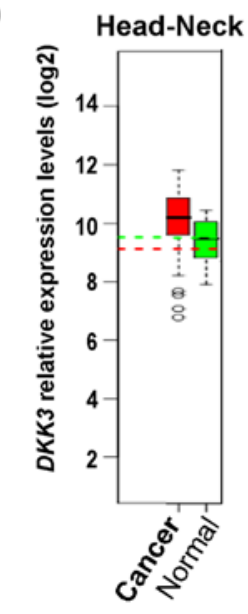

Figure 1. Analysis of DKK3 mRNA expression in OSCC and HNSCC using Oncomine and GENT database. Box-plot diagrams were analyzed to compare the DKK3 mRNA levels in normal oral, tongue and buccal mucosa tissue with that in (A) OSCC, (B) TSCC and (C) HNSCC using the Oncomine dataset. The vertical axis represents the $\log 2$ median value. The upper (75\%) and lower $(25 \%)$ quartiles are represented by the upper and lower borders of the boxes, respectively. (D) DKK3 mRNA expression levels in HNSCC and normal head-neck tissues in the GENT database.

A

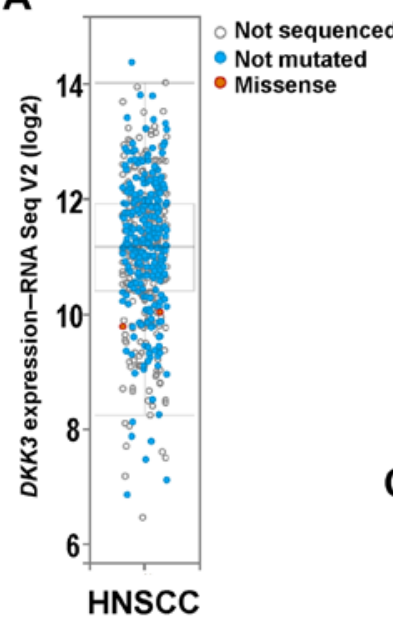

B

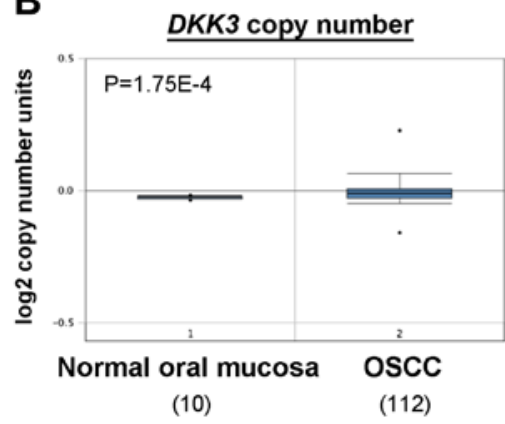

C

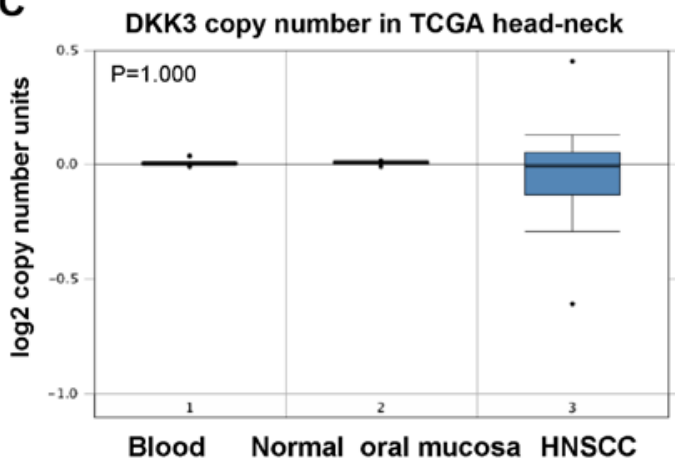

(74)

(290)

Figure 2. DKK3 gene mutation and copy number analysis in OSCC and HNSCC. (A) DKK3 expression and mutation status from the TCGA HNSCC cohorts. (B) DKK 3 copy number analysis in OSCC using the Oncomine dataset. (C) DKK3 copy number analysis in the TCGA Head-Neck cohort using the Oncomine database. 
Table I. DKK3 expression in the carcinogenesis of OSF.

\begin{tabular}{|c|c|c|c|c|c|c|c|}
\hline \multirow[b]{2}{*}{ Group } & \multirow[b]{2}{*}{$\mathrm{n}$} & \multicolumn{4}{|c|}{ DKK3 } & \multirow{2}{*}{$\begin{array}{c}\text { DKK3 expression } \\
(\%)\end{array}$} & \multirow{2}{*}{$\begin{array}{c}\text { Mean DKK3 } \\
\text { score }\end{array}$} \\
\hline & & - & + & ++ & +++ & & \\
\hline Normal & 15 & 9 & 3 & 2 & 1 & 33.33 & 1.73 \\
\hline OSF & 45 & 10 & 12 & 12 & 11 & 77.78 & 3.73 \\
\hline $\mathrm{E}$ & 15 & 4 & 3 & 4 & 4 & 73.33 & 3.53 \\
\hline $\mathrm{M}$ & 15 & 3 & 4 & 4 & 4 & 80.00 & 3.67 \\
\hline A & 15 & 3 & 2 & 5 & 5 & 80.00 & 4.00 \\
\hline OSCC & 55 & 2 & 17 & 17 & 19 & 96.36 & 4.45 \\
\hline
\end{tabular}

DKK3, dickkopf WNT signaling pathway inhibitor 3. OSF, oral submucous fibrosis: E, early stage; M, moderately advanced stage; A, advanced stage. OSCC, oral squamous cell carcinoma.

Table II. Correlation of DKK3 expression and the clinicopathological features of the OSCC cases.

\begin{tabular}{lcccc}
\hline & & \multicolumn{2}{c}{ DKK3 } & \\
$\begin{array}{l}\text { Clinicopathological } \\
\text { features }\end{array}$ & $\begin{array}{c}\text { Total } \\
\text { (n) }\end{array}$ & + & - & P-value \\
\hline Age (years) & & & & $>0.05$ \\
$\quad<50$ & 43 & 42 & 1 & \\
$\geq 50$ & 12 & 11 & 1 & \\
Gender & & & & $<0.01$ \\
Male & 52 & 52 & 0 & \\
Female & 3 & 1 & 2 & \\
Tumor site & & & & $>0.05$ \\
Tongue & 40 & 39 & 1 & \\
Others & 15 & 13 & 2 & \\
Primary tumor & & & & $>0.05$ \\
T1+T2 & 12 & 11 & 1 & \\
T3+T4 & 43 & 42 & 1 & \\
TNM stage & & & & $>0.05$ \\
I+II & 12 & 11 & 1 & \\
III+IV & 43 & 42 & 1 & \\
Differentiation grade & & & & $>0.05$ \\
Well & 20 & 19 & 1 & \\
Moderate-poor & 35 & 34 & 1 & \\
\hline
\end{tabular}

DKK3, dickkopf WNT signaling pathway inhibitor 3; OSCC, oral squamous cell carcinoma.

staining using a DKK3-specific antibody in normal oral mucous, OSF and OSCC tissues. Six of 15 (33.3\%) normal oral mucous cases showed DKK3 positivity in the cytoplasm, and 35 of 45 (77.8\%) OSF tissues showed cytoplasmic DKK3 expression, including tissues from 11 of $15(73.3 \%)$ early stage, 12 of $15(80.0 \%)$ moderately advanced stage and 12 of $15(80.0 \%)$ advanced stage, as well as 53 of 55 (96.4\%) OSCC (Fig. 3A). The average values of DKK 3 expression varied in the different tissue samples, including the mean score of 1.73 in normal oral mucosa tissues, 3.73 in OSF tissues and 4.45 in OSCC tissues (Fig. 3B; Table I). DKK3 expression level was gradually increased in normal oral, OSF and OSCC tissues $(\mathrm{P}<0.05)$.

The correlation between DKK3 expression and the clinicopathological features of the OSCC cases was analyzed, including age, gender, tumor site, primary tumor, TNM stage and differentiation grade. DKK3 expression between male patients and female patients was statistically significant (Table II). These data suggest that DKK3 is upregulated at the protein level in the carcinogenesis of OSF.

DKK3 mRNA expression is elevated in the carcinogenesis of $O S F . D K K 3$ expression at the mRNA level was examined in normal oral mucosa and OSF tissues, as well as OSCC and their paired adjacent tissues by semi-quantitative RT-PCR. We found that $D K K 3$ was expressed in normal oral mucosa (Fig. 4A) and OSF tissues in different stages (Fig. 4B) with varied levels. $D K K 3$ expression was detected in OSCC and their adjacent OSF tissues. Results showed that $D K K 3$ expression levels were higher in OSCC tissues, than their adjacent OSF tissues (Fig. 4C). Moreover, the DKK3 expression level was upregulated in OSCC tissues, compared with the paired adjacent normal tissues (Fig. 4D). Increased mRNA expression level of $D K K 3$ in OSCC tissues was further confirmed by real-time RT-PCR, compared with their adjacent normal or OSF tissues (Fig. 4E). Therefore, the DKK3 mRNA expression level is increased in the carcinogenesis of OSF.

Analysis of co-expressed genes with DKK3 in OSCC. To further investigate the potential function of DKK3 in OSCC, average linkage hierarchical clustering was applied to identify the co-expressed gene set with DKK3 in the Oncomine database. One study comparing DKK3 expression data for normal oral mucosa and OSCC tissues was selected for analysis, by using human genome U133A array with 12,624 genes measured. Nineteen genes were co-expressed with DKK3 in OSCC with correlation rate from 0.803 to 0.954 (Fig. 5): TSPYL5, MNDA, ZNF415, NEFH, PCDH7, TMEM38B, HSPB11, BAG2, LGR5, co10orf137, PMPCB, GUF1, RPAP3, IGKV3-20, IGL, IGHM, CARS2, RYR3 and ZNF107. Several of these genes are related to Wnt and p53 signaling, apoptosis, $\mathrm{Ca}^{2+}$ and mitochondrial signaling pathways. 
A

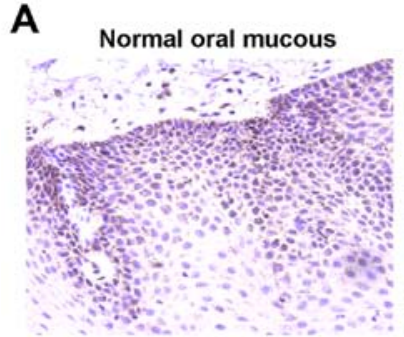

OSF-M

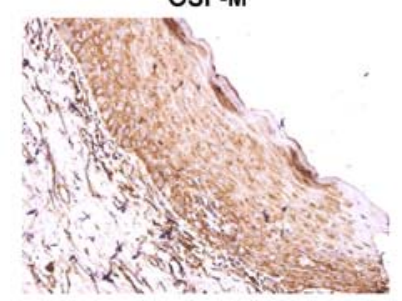

OSCC

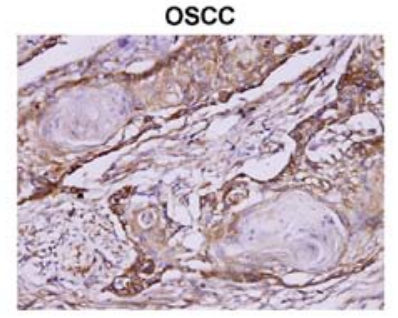

OSF-E

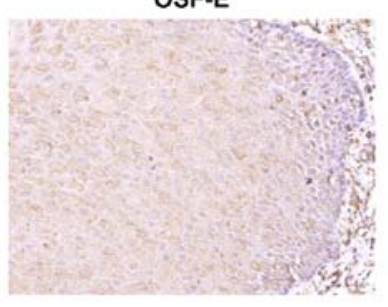

OSF-A

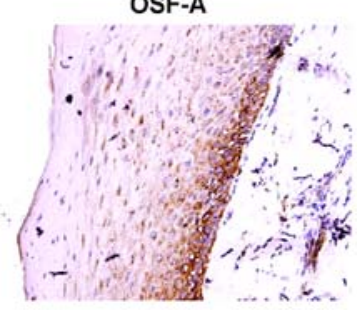

B

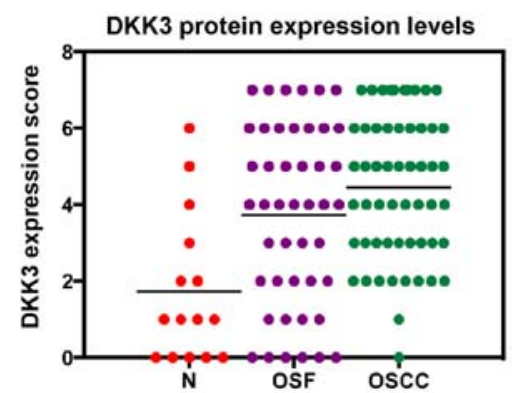

Figure 3. Immunohistochemical staining of DKK3 in normal oral mucosa, OSF and OSCC tissues. (A) Normal oral tissues exhibited weak DKK3 protein expression in the cytoplasm. OSF early (E), moderately advanced (M), and advanced (A) stage tissues showed DKK3-positive expression in cytoplasmic parts. Primary OSCC showed very strongly cytoplasmic immunoreaction for DKK3. Original magnification, $\mathrm{x} 400$. (B) The average values of DKK3 expression in the different tissue samples. N, normal oral tissues; OSF, oral submucous fibrosis; OSCC, oral squamous cell carcinoma; DKK3, dickkopf WNT signaling pathway inhibitor 3 .

\section{Discussion}

Cumulative genetic and epigenetic aberrations in cancer genes contribute to OSCC tumorigenesis. Thus, identification of key genes involved in OSCC initiation and progression, particularly malignant progression of precancerous OSF lesions, is vital. As one of the Wnt antagonists, DKK family members antagonize the Wnt signaling pathway by interacting with the Wnt co-receptors LRP5 and LRP6, further preventing their interaction with Wnt ligands $(10,22,23)$. Unlike other family members, only DKK3 and DKKL1 contain sgy-domain thus are divergent members of the DKK family (10), suggesting possible Wnt-independent function of DKK3 other than as a Wnt antagonist in tumorigenesis.

DKK3 downregulation by promoter CpG methylation has been reported in multiple types of cancer, including thyroid (24), lung $(25,26)$, gastric (27), colon (27), hepatocellular $(27,28)$, breast $(29,30)$, ovarian (31), cervical (32) and renal $(33,34)$ cancers, as well as acute myeloid leukemia $(35)$ and glioma (36). Various studies found that DKK3 is a putative Wnt signaling inhibitor. In OSCC, DKK3 possesses tumorsuppressive function or oncogenic function. DKK3 was found to be epigenetically inactivated in oral cancer, along with other Wnt antagonists WIF1 and SFRPs (12). DKK3 deletion was detected in HNSCC samples using Oncomine and TCGA databases, although no statistical significance and significantly prolonged overall survival were observed, suggesting that DKK3 may function as a tumor-suppressor gene (TSG) in OSCC. Recent studies provide strong evidence that DKK3 plays an oncogenic role in OSCC. DKK3 protein expression was found to be increased in dysplasia and further in OSCC, and was found to be correlated with OSCC metastasis and poor prognosis, independent of the Wnt signaling pathway (13-15).

In the present study, we found that DKK3 was statistically significantly upregulated in OSCC development by bioinformatic analysis. A rare genetic mutation of DKK3 was found in OSCC. DKK3 gene copy number was increased in OSCC, compared to normal oral mucosa, thus further causing DKK3 upregulation in OSCC. We also found that DKK3 either at the protein level or at the mRNA level was expressed in normal oral mucosal tissues, and the levels were gradually increased in the different stages of OSF and OSCC tissues with BQ chewing, which is consistent with other reports. By analyzing co-expressed genes with DKK3, potential biological functions and molecular mechanisms of DKK3 in OSCC pathogenesis were shown. For example, DKK3 activates the Wnt signaling pathway by binding to and activating LGR5 (37,38); DKK3 was found to inhibit the p53 signaling pathway by modulating TSPYL5 (39) and ZNF415 (40); DKK3 also suppressed apoptosis through the mitochondrial signaling pathway.

In summary, we provide evidence that the DKK3 expression level is increased in the carcinogenesis of OSF. DKK3 copy number gains are responsible for its upregulation in OSCC. The present study revealed the oncogenic role of DKK3 in the malignant progression of OSF, and sheds light on the development of a valuable tumor biomarker for the early detection of OSCC. 
A

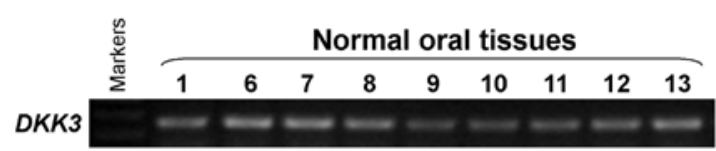

B

\begin{tabular}{|lll} 
OSF \\
$\stackrel{24}{E}$ & $\frac{28}{M}$ & $\frac{26}{A}$ \\
\hline & & $=$
\end{tabular}

C OSCC and paired adjacent OSF tissues

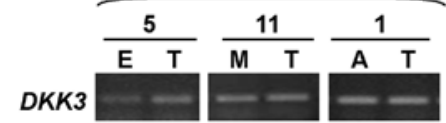

D OSCC and paired adjacent normal tissues

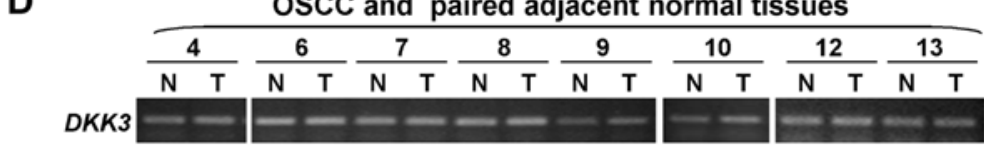

E

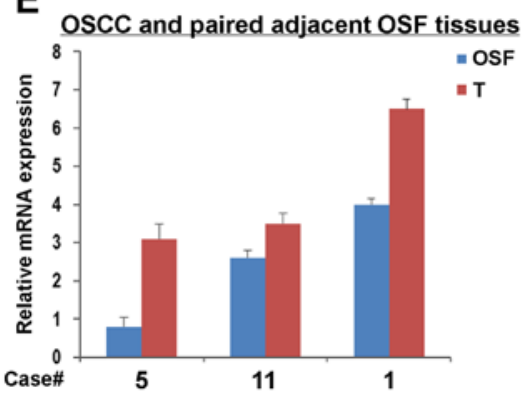

OSCC and paired adjacent normal tissues

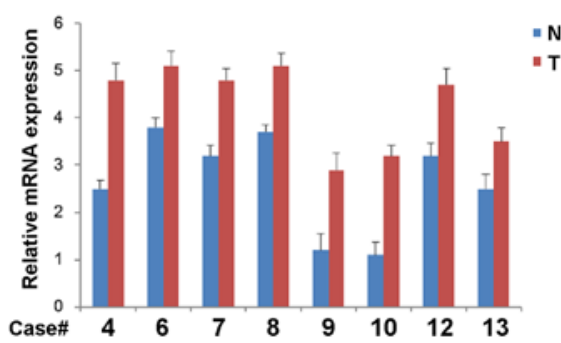

Figure 4. Detection of $D K K 3$ mRNA expression in normal oral mucosa, OSF and OSCC tissues. Semi-quantitative RT-PCR assessed $D K K 3$ expression in (A) normal oral mucosa tissues, (B) OSF tissues, (C) OSCC and paired adjacent OSF tissues, as well as (D) OSCC and paired adjacent normal tissues. GAPDH was used as an internal control. (E) Quantitative real-time RT-PCR was used to confirm DKK3 expression in samples from OSCC and paired adjacent OSF or normal tissues. DKK3, dickkopf WNT signaling pathway inhibitor 3. OSF, oral submucous fibrosis: E, early stage; M, moderately advanced stage; A, advanced stage. OSCC, oral squamous cell carcinoma (T); N, normal mucosa tissue.

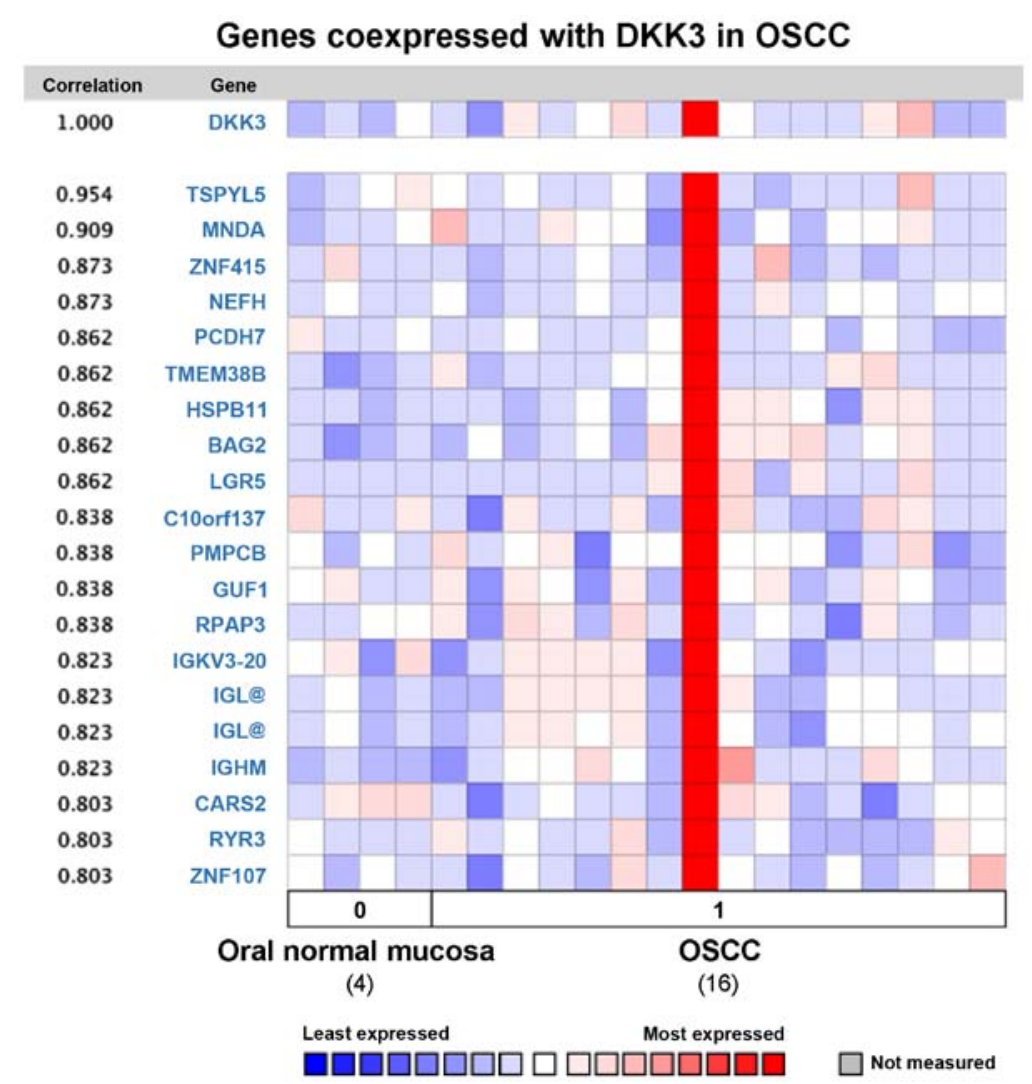

Figure 5. Co-expression gene analysis of DKK3 in OSCC using the Oncomine database. Heat map of the co-expression profile of DKK3 in OSCC tissues, compared with oral normal mucosa tissues. DKK3, dickkopf WNT signaling pathway inhibitor 3; OSCC, oral squamous cell carcinoma. 


\section{Acknowledgements}

The present study was supported by the National Natural Science Foundation of China (no. 81202133) and the Cross Research Fund of Biomedical Engineering of Shanghai Jiaotong University (YG2016MS04).

\section{References}

1. Torre LA, Bray F, Siegel RL, Ferlay J, Lortet-Tieulent J and Jemal A: Global cancer statistics, 2012. CA Cancer J Clin 65: 87-108, 2015

2. Braakhuis BJ, Brakenhoff RH and Leemans CR: Head and neck cancer: Molecular carcinogenesis. Ann Oncol 16 (Suppl 2): ii249-ii250, 2005.

3. Leemans CR, Braakhuis BJ and Brakenhoff RH: The molecular biology of head and neck cancer. Nat Rev Cancer 11: 9-22, 2011.

4. Choi S and Myers JN: Molecular pathogenesis of oral squamous cell carcinoma: Implications for therapy. J Dent Res 87: 14-32, 2008.

5. Pindborg JJ, Murti PR, Bhonsle RB, Gupta PC, Daftary DK and Mehta FS: Oral submucous fibrosis as a precancerous condition. Scand J Dent Res 92: 224-229, 1984.

6. Tilakaratne WM, Klinikowski MF, Saku T, Peters TJ and Warnakulasuriya S: Oral submucous fibrosis: Review on aetiology and pathogenesis. Oral Oncol 42: 561-568, 2006.

7. Wollina U, Verma SB, Ali FM and Patil K: Oral submucous fibrosis: An update. Clin Cosmet Investig Dermatol 8: 193-204, 2015.

8. Polakis P: Wnt signaling in cancer. Cold Spring Harb Perspect Biol 4: 4, 2012

9. Veeck $\mathbf{J}$ and Dahl E: Targeting the Wnt pathway in cancer: The emerging role of Dickkopf-3. Biochim Biophys Acta 1825: 18-28, 2012.

10. Niehrs C: Function and biological roles of the Dickkopf family of Wnt modulators. Oncogene 25: 7469-7481, 2006

11. Katase N, Gunduz M, Beder L, Gunduz E, Lefeuvre M, Hatipoglu OF, Borkosky SS, Tamamura R, Tominaga S, Yamanaka N, et al: Deletion at Dickkopf (dkk)-3 locus (11p15.2) is related with lower lymph node metastasis and better prognosis in head and neck squamous cell carcinomas. Oncol Res 17: 273-282, 2008

12. Pannone G, Bufo P, Santoro A, Franco R, Aquino G, Longo F, Botti G, Serpico R,Cafarelli B, Abbruzzese A, et al: WNT pathway in oral cancer: Epigenetic inactivation of WNT-inhibitors. Oncol Rep 24: 1035-1041, 2010.

13. Fujii M, Katase N, Lefeuvre M, Gunduz M, Buery RR, Tamamura R, Tsujigiwa H and Nagatsuka H: Dickkopf (Dkk)-3 and $\beta$-catenin expressions increased in the transition from norma oral mucosal to oral squamous cell carcinoma. J Mol Histol 42: 499-504, 2011.

14. Kataoka K, Du G, Maehara N, Murata H, Sakaguchi M and Huh N: Expression pattern of REIC/Dkk-3 in mouse squamous epithelia. Clin Exp Dermatol 37: 428-431, 2012.

15. Katase N, Lefeuvre M, Tsujigiwa H, Fujii M, Ito S, Tamamura R, Buery RR, Gunduz M and Nagatsuka H: Knockdown of Dkk-3 decreases cancer cell migration and invasion independently of the Wnt pathways in oral squamous cell carcinoma-derived cells. Oncol Rep 29: 1349-1355, 2013.

16. Gupta PC, Sinor PN, Bhonsle RB, Pawar VS and Mehta HC: Oral submucous fibrosis in India: A new epidemic? Natl Med J India 11: 113-116, 1998.

17. Zhou S, Chen L, Mashrah M, Zhu Y, He Z, Hu Y, Xiang T, Yao Z, Guo $\mathrm{F}$ and Zhang C: Expression and promoter methylation of Wnt inhibitory factor-1 in the development of oral submucous fibrosis. Oncol Rep 34: 2636-2642, 2015.

18. Zhou S, Chen L, Mashrah M, Zhu Y, Liu J, Yang X, He Z, Wang L, Xiang T, Yao Z, et al: Deregulation of secreted frizzledrelated proteins is associated with aberrant $\beta$-catenin activation in the carcinogenesis of oral submucous fibrosis. Onco Targets Ther 8: 2923-2931, 2015.

19. Peng CH, Liao CT, Peng SC, Chen YJ, Cheng AJ, Juang JL, Tsai CY, Chen TC, Chuang YJ, Tang CY, et al: A novel molecular signature identified by systems genetics approach predicts prognosis in oral squamous cell carcinoma. PLoS One 6: e23452, 2011.

20. Ye H, Yu T, Temam S, Ziober BL, Wang J, Schwartz JL, Mao L, Wong DT and Zhou X: Transcriptomic dissection of tongue squamous cell carcinoma. BMC Genomics 9: 69, 2008.
21. Ginos MA, Page GP, Michalowicz BS, Patel KJ, Volker SE, Pambuccian SE, Ondrey FG, Adams GL and Gaffney PM: Identification of a gene expression signature associated with recurrent disease in squamous cell carcinoma of the head and neck. Cancer Res 64: 55-63, 2004.

22. Holmen SL, Robertson SA, Zylstra CR and Williams BO: Wnt-independent activation of beta-catenin mediated by a Dkk1-Fz5 fusion protein. Biochem Biophys Res Commun 328: 533-539, 2005.

23. Fujii Y, Hoshino T and Kumon H: Molecular simulation analysis of the structure complex of C2 domains of DKK family members and $\beta$-propeller domains of LRP5/6: Explaining why DKK3 does not bind to LRP5/6. Acta Med Okayama 68: 63-78, 2014.

24. Yin DT, Wu W, Li M, Wang QE, Li H, Wang Y, Tang Y and Xing M: $D K K 3$ is a potential tumor suppressor gene in papillary thyroid carcinoma. Endocr Relat Cancer 20: 507-514, 2013.

25. Jung IL, Kang HJ, Kim KC and Kim IG: Knockdown of the Dickkopf 3 gene induces apoptosis in a lung adenocarcinoma. Int J Mol Med 26: 33-38, 2010.

26. Kobayashi K, Ouchida M, Tsuji T, Hanafusa H, Miyazaki M, Namba M, Shimizu N and Shimizu K: Reduced expression of the REIC/Dkk-3 gene by promoter-hypermethylation in human tumor cells. Gene 282: 151-158, 2002.

27. Sato H, Suzuki H, Toyota M, Nojima M, Maruyama R, Sasaki S, Takagi H, Sogabe Y, Sasaki Y, Idogawa M, et al: Frequent epigenetic inactivation of $D I C K K O P F$ family genes in human gastrointestinal tumors. Carcinogenesis 28: 2459-2466, 2007.

28. Liang L, He H, Lv R, Zhang M, Huang H, An Z and Li S: Preliminary mechanism on the methylation modification of Dkk-1 and Dkk-3 in hepatocellular carcinoma. Tumour Biol 36: 1245-1250, 2015

29. Veeck J, Bektas N, Hartmann A, Kristiansen G, Heindrichs U, Knüchel R and Dahl E: Wnt signalling in human breast cancer: Expression of the putative Wnt inhibitor Dickkopf-3 (DKK3) is frequently suppressed by promoter hypermethylation in mammary tumours. Breast Cancer Res 10: R82, 2008.

30. Xiang T, Li L, Yin X, Zhong L, Peng W, Qiu Z, Ren G and Tao Q: Epigenetic silencing of the WNT antagonist Dickkopf 3 disrupts normal $\mathrm{Wnt} / \beta$-catenin signalling and apoptosis regulation in breast cancer cells. J Cell Mol Med 17: 1236-1246, 2013.

31. You A, Fokas E, Wang LF, He H, Kleb B, Niederacher D, Engenhart-Cabillic R and An HX: Expression of the Wnt antagonist DKK3 is frequently suppressed in sporadic epithelial ovarian cancer. J Cancer Res Clin Oncol 137: 621-627, 2011.

32. Lee EJ, Jo M, Rho SB, Park K, Yoo YN, Park J, Chae M, Zhang W and Lee JH: $D k k 3$, downregulated in cervical cancer, functions as a negative regulator of beta-catenin. Int J Cancer 124: 287-297, 2009.

33. Kurose K, Sakaguchi M, Nasu Y, Ebara S, Kaku H, Kariyama R, Arao Y, Miyazaki M, Tsushima T, Namba M, et al: Decreased expression of REIC/Dkk-3 in human renal clear cell carcinoma. J Urol 171: 1314-1318, 2004.

34. Ueno K, Hirata H, Majid S, Chen Y, Zaman MS, Tabatabai ZL, Hinoda Y and Dahiya R: Wnt antagonist DICKKOPF-3 (Dkk-3) induces apoptosis in human renal cell carcinoma. Mol Carcinog 50: 449-457, 2011.

35. Valencia A, Román-Gómez J, Cervera J, Such E, Barragán E, Bolufer P, Moscardó F, Sanz GF and Sanz MA: Wnt signaling pathway is epigenetically regulated by methylation of Wnt antagonists in acute myeloid leukemia. Leukemia 23: 1658-1666, 2009.

36. Mizobuchi Y, Matsuzaki K, Kuwayama K, Kitazato K, Mure H, Kageji T and Nagahiro S: REIC/Dkk-3 induces cell death in human malignant glioma. Neuro Oncol 10: 244-253, 2008.

37. Carmon KS, Lin Q, Gong X, Thomas A and Liu Q: LGR5 interacts and cointernalizes with Wnt receptors to modulate Wnt/ $\beta$-catenin signaling. Mol Cell Biol 32: 2054-2064, 2012.

38. de Lau W, Barker N, Low TY, Koo BK, Li VS, Teunissen H, Kujala P, Haegebarth A, Peters PJ, van de Wetering M, et al: Lgr5 homologues associate with Wnt receptors and mediate R-spondin signalling. Nature 476: 293-297, 2011.

39. Epping MT, Meijer LA, Krijgsman O, Bos JL, Pandolfi PP and Bernards R: TSPYL5 suppresses p53 levels and function by physical interaction with USP7. Nat Cell Biol 13: 102-108, 2011.

40. Cheng Y, Wang Y, Li Y, Deng Y, Hu J, Mo X, Li N, Li Y, Luo N, Yuan W, et al: A novel human gene ZNF415 with five isoforms inhibits AP-1- and p53-mediated transcriptional activity. Biochem Biophys Res Commun 351: 33-39, 2006. 